Supplement of Hydrol. Earth Syst. Sci., 25, 4741-4758, 2021

https://doi.org/10.5194/hess-25-4741-2021-supplement

(c) Author(s) 2021. CC BY 4.0 License.

(c) (i)

Supplement of

\title{
Assessing the large-scale plant-water relations in the humid, subtropical Pearl River basin of China
}

\section{Hailong Wang et al.}

Correspondence to: Hailong Wang (wanghlong3@mail.sysu.edu.cn,wh184@ hotmail.com)

The copyright of individual parts of the supplement might differ from the article licence. 


\section{Supplementary materials}

The materials in this document are provided to support the data selection for the analysis in the manuscript. They include comparisons of

(1) Precipitation (P) data from GLDAS_Noah v2.1 and from national standard meteorological stations obtained from the China Meteorological Administration;

(2) Potential evapotranspiration (ETp) from GLDAS_Noah v2.1 and MOD16A2;

(3) Gross Primary Production (GPP) from MOD17A2, VPM and PML v2.

Comparisons of these data spatially and temporally are intended to provide an overview of the uncertainty among different datasets with different algorithms, and further to support the analysis based on the ensemble means of multiple datasets for each variable under the assumption that the ensemble means can reduce the data errors. We area aware that it is difficult to conclude which individual dataset is more accurate than its counterparts without extensive gridded measurements on the ground.

\section{- Comparison of P between GLDAS and observation}
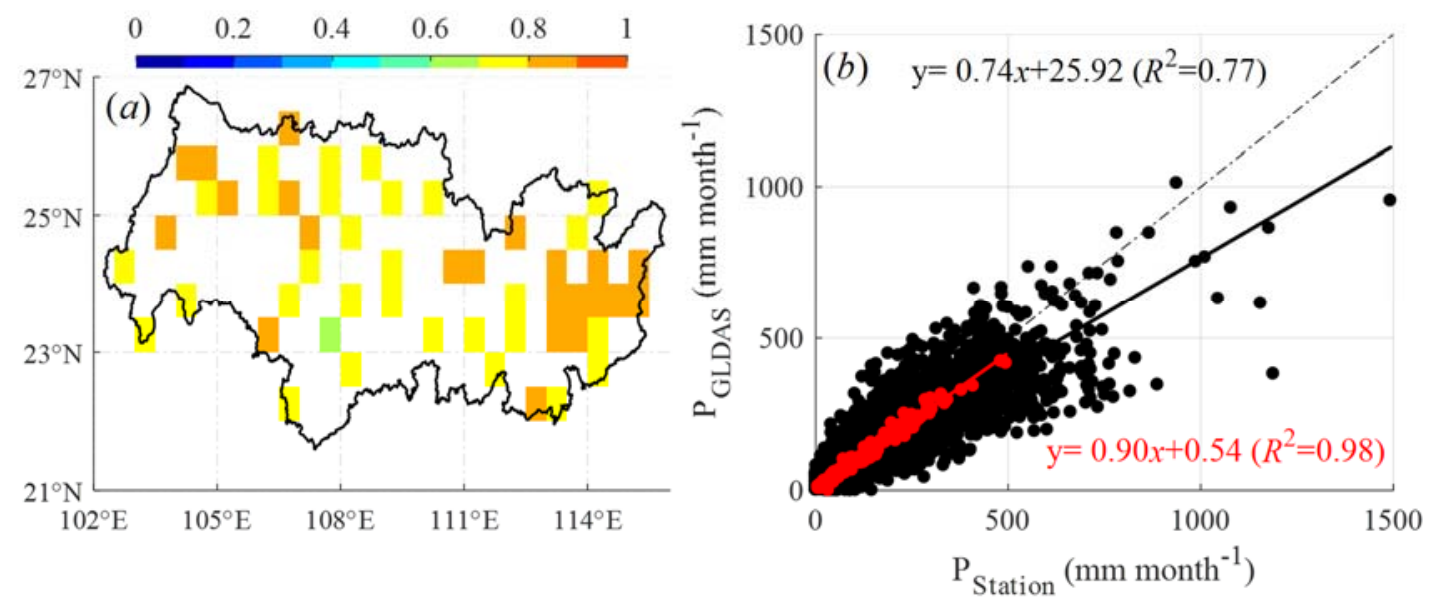

Figure S1. Comparison of monthly precipitation (P) between GLDAS and the national meteorological stations. (a) Spatial distribution of coefficient of determination $\left(R^{2}\right)$ over the pixels with available stations; and (b) Scatter plot of monthly P for all valid pixels (in black) and the average monthly $\mathrm{P}$ over the valid pixels (in red).

Fig. S1 shows that monthly precipitation data from GLDAS and meteorological stations agree well both spatially and temporally. The spatial coefficients of determination $\left(R^{2}\right)$ range from 0.7 to 0.9 in pixels where stations are available, and the temporal $R^{2}$ is 0.77 
(with a regression slope of 0.74 ) for all data in all valid pixels. Over the entire basin, the regression slope is 0.90 between station-based and GLDAS values. The comparison indicates that the gridded GLDAS precipitation data can be used to analyze the dynamics and relationships of hydroclimate and vegetation parameters. This would give advantage of GLDAS for long term spatiotemporal studies.

\section{- Comparison of ETp between GLDAS and MOD16}

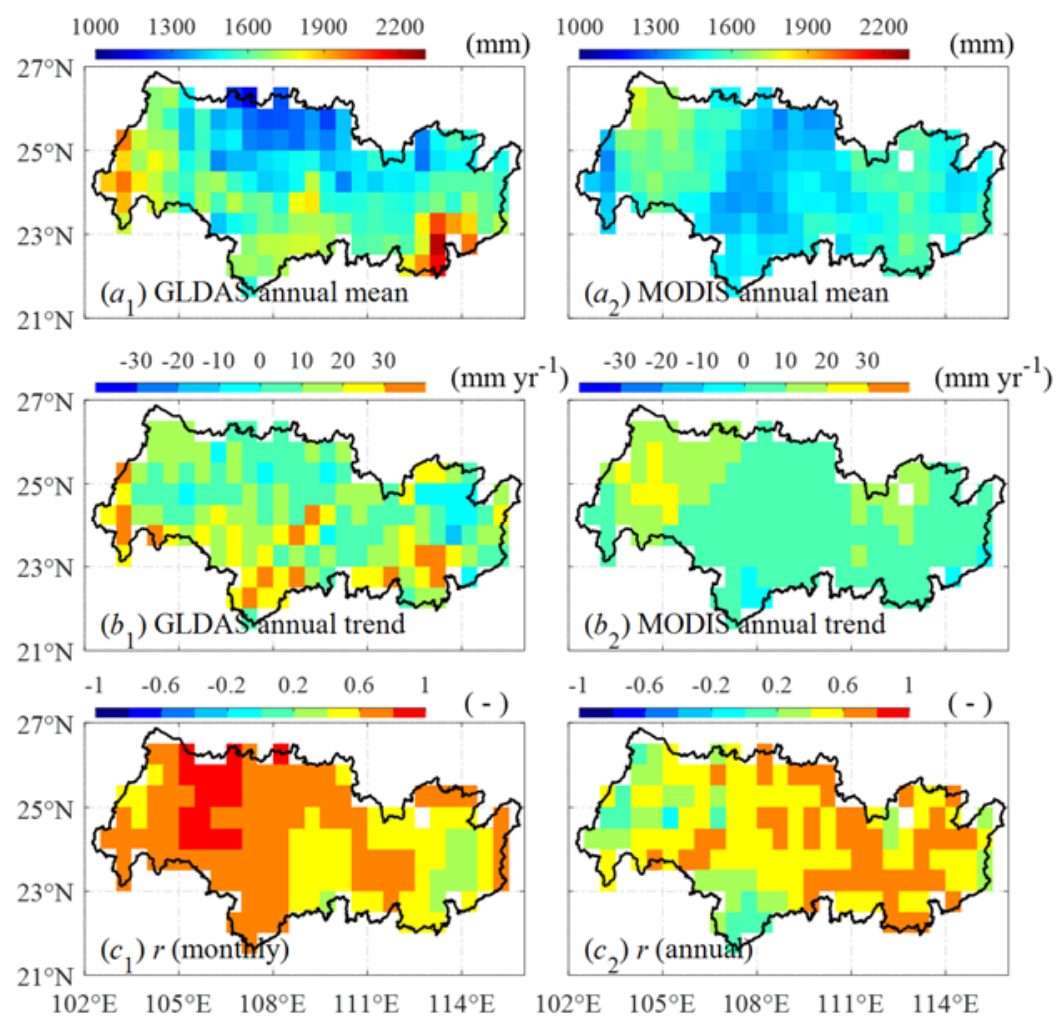

Figure S2. $\left(a_{1}-a_{2}\right)$ Annual mean ETp from GLDAS and MODIS; $\left(b_{1}-b_{2}\right)$ annual trend given by the MK test method for ETp from GLDAS and MODIS; $\left(c_{1}-c_{2}\right)$ correlation coefficient between ETp from GLDAS and MODIS at the monthly and annual scale, respectively.

Fig. S2 shows that GLDAS gave overall slightly higher ETp than MODIS, and the spatial patterns are different that GLDAS shows higher ETp in the south and lower ETp in the north of the basin, while MODIS shows that lower ETp appears in the middle and higher ETp in the east and west of the basin. Both products show ETp has been increasing over the 13 years, although GLDAS gives greater increase rates. GLDAS shows that ETp increase was largest over the croplands in the middle-south of the basin. Spatially, the correlation coefficient between these two datasets ranges from 0.26 to 0.87 at the monthly scale with most within $0.6 \sim 0.9$, and ranges from -0.11 to 0.76 at the annual scale with most within $0.4 \sim 0.8$. 

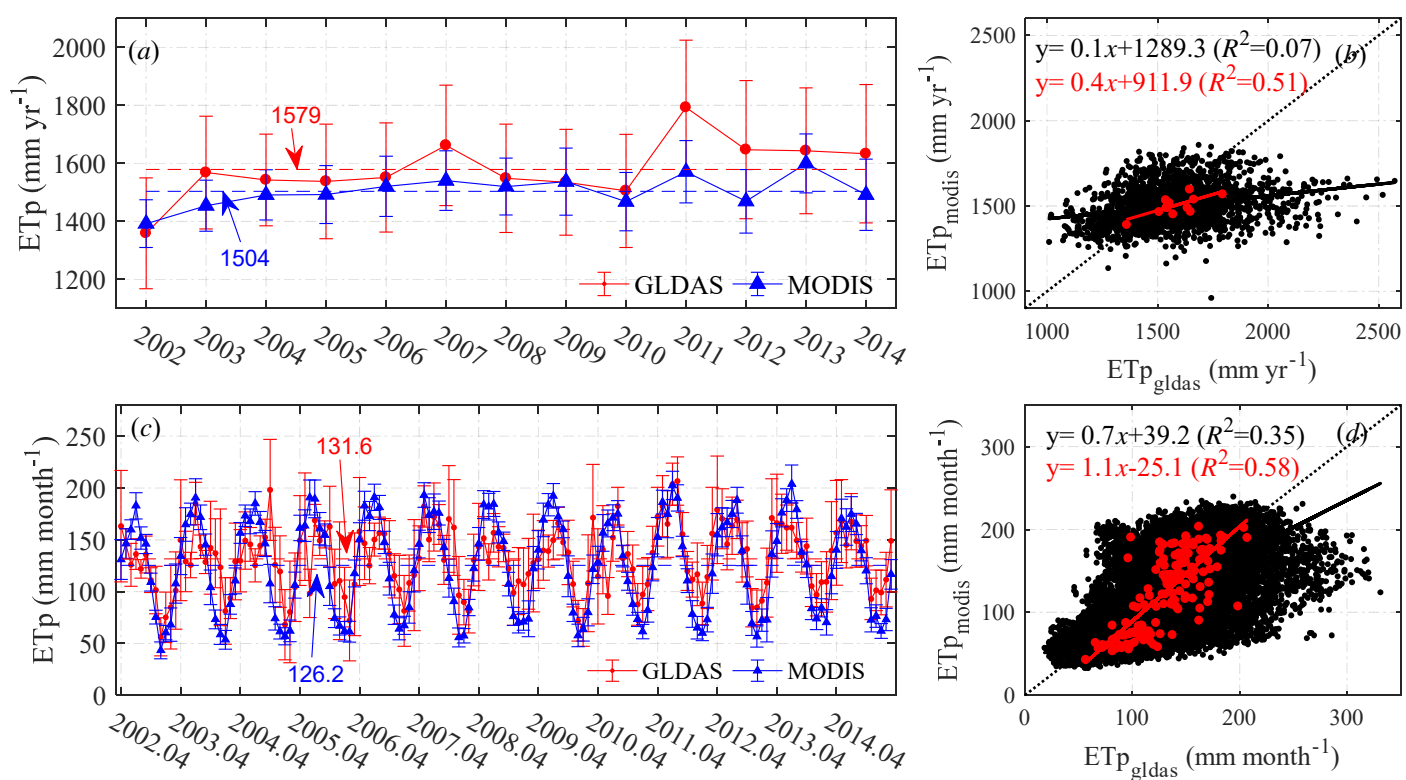

Figure S3. $(a, c)$ Variations of the mean annual and monthly ETp from GLDAS and MODIS over the entire basin. Dashed lines are annual and monthly means for the two ETp series; $(b, d)$ Scatter plot of annual/monthly ETp for all pixels (in black) and the average annual/monthly ETp over the entire basin (in red).

Temporally, ETp from GLDAS has higher values than that from MODIS (Fig. S3). The average annual ETp from GLDAS is $1579 \pm 102$ and $1504 \pm 54 \mathrm{~mm} \mathrm{yr}^{-1}$, and the average monthly ETp is $132 \pm 30$ and $125 \pm 46 \mathrm{~mm} \mathrm{month}^{-1}$. The coefficient of determination between the two sources is 0.58 and 0.51 at the monthly and annual scale over the entire basin, with a regression slope of 1.1 and 0.4 , respectively. When considering data of all pixels, the GLDAS data is much higher than MODIS. This infers that the data uncertainty accumulates from short to long period. In lack of ground truth data, and to reduce the effect of errors embedded in each individual product, we calculated the average ETp from the two sources for later analysis.

\section{- Comparison of GPP between MOD17, VPM and PML}



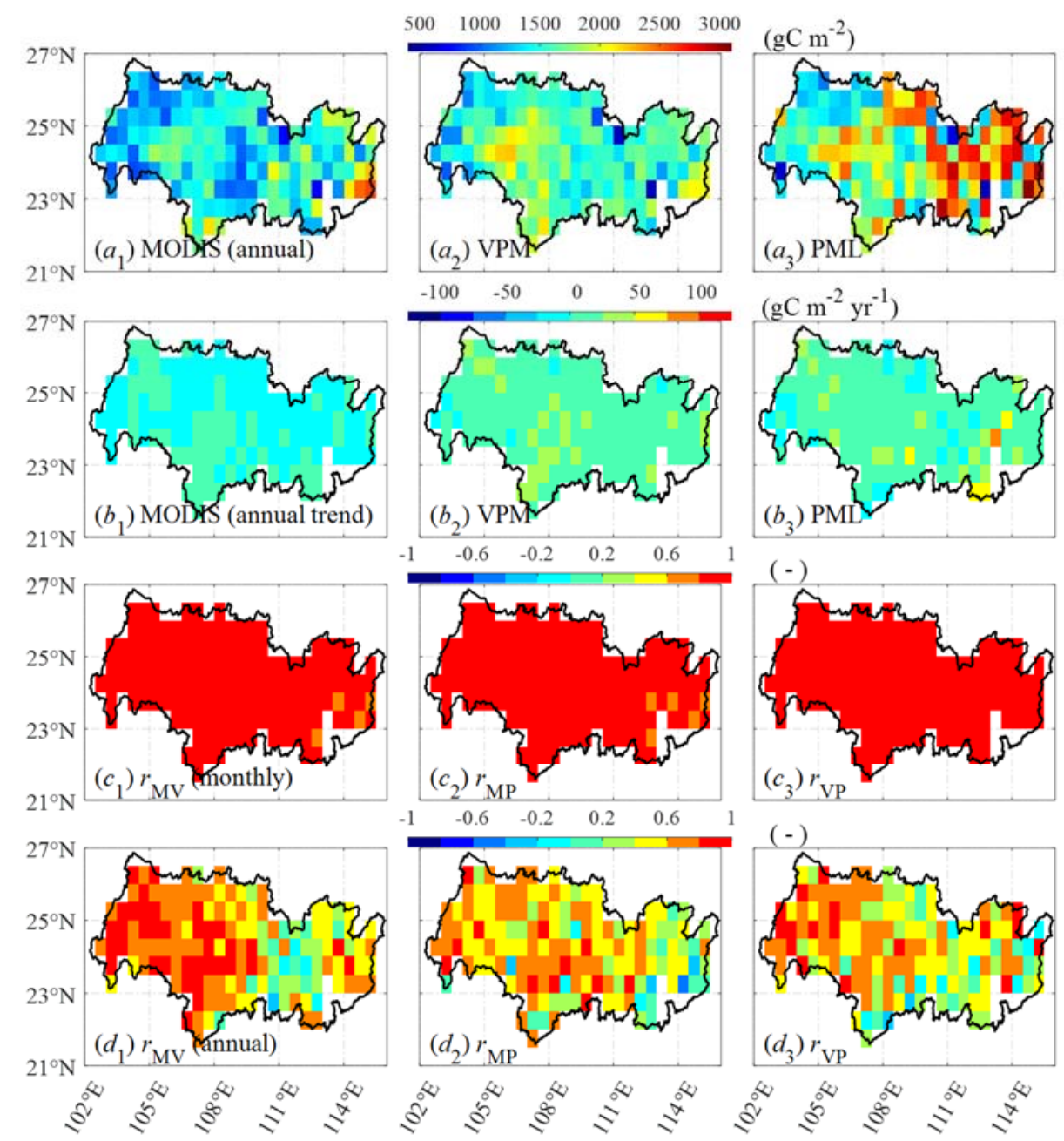

Figure S4. Comparison of GPP data from different sources based on different algorithms. $\left(a_{1-3}\right)$ mean annual GPP from MODIS, VPM and PML; $\left(b_{1-3}\right)$ Trends of annual GPP; $\left(c_{1-3} \& d_{1-3}\right)$ Correlation coefficients between GPP from MODIS and VPM $\left(r_{\mathrm{MV}}\right)$, MODIS and PML $\left(r_{\mathrm{MP}}\right)$ and from VPM and PML $\left(r_{\mathrm{VP}}\right)$ at the monthly and annual scales, respectively.

The pattern between MODIS and VPM is similar and differs from PML where the latter shows higher GPP values spatially (Fig. S4). The annual trend for majority of the basin lies in the range of -25 to $25 \mathrm{gC} \mathrm{m}^{2} \mathrm{yr}^{-1}$. Regardless of the different values, GPP from the three sources agree well with correlation coefficient $r>0.9$ in most pixels. 

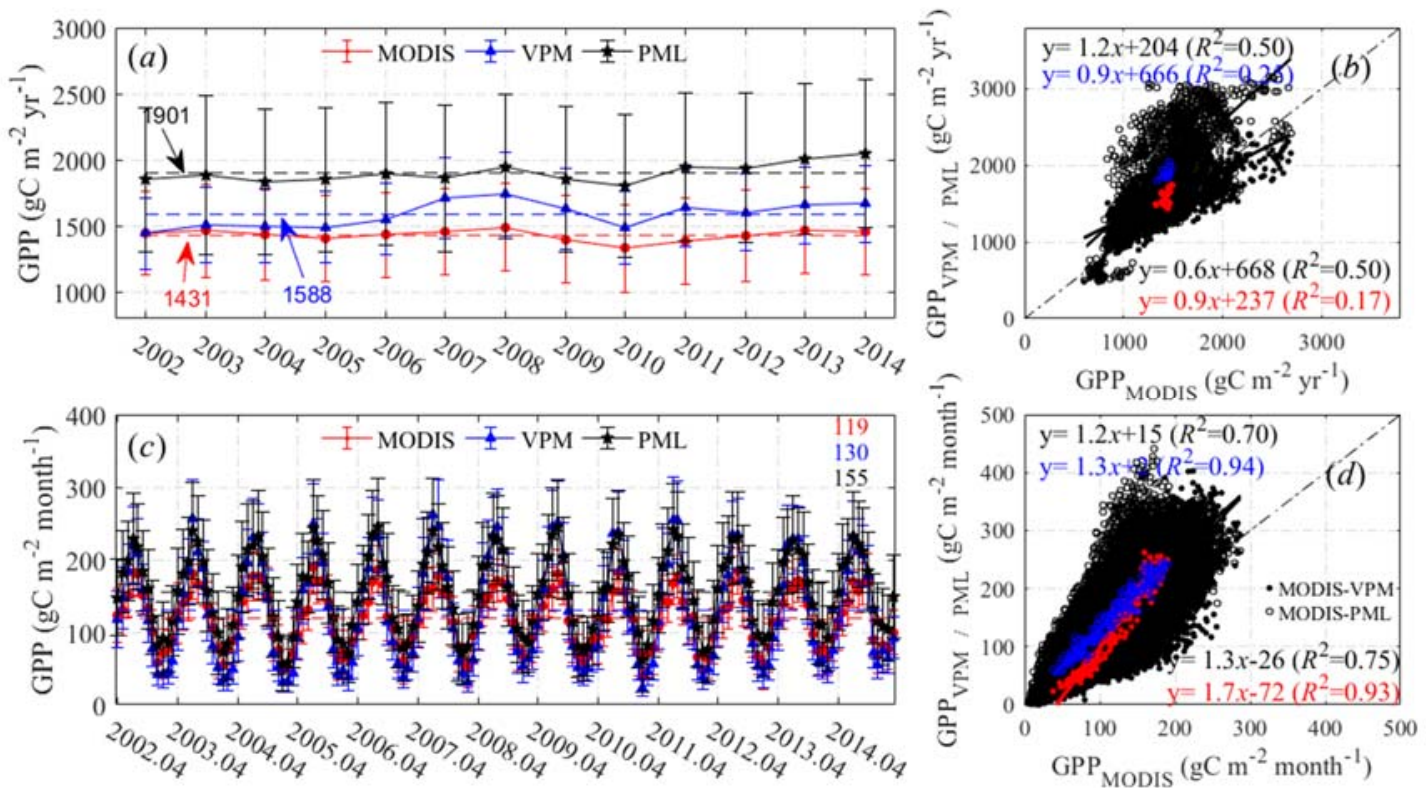

Figure S5. $(a, c)$ Variations of the mean annual and monthly GPP from MODIS, VPM and PML over the entire basin. Dashed lines are annual and monthly means for the three GPP series; ( $b$, d) Scatter plot of the annual/monthly GPP from MODIS versus VPM and PML over all pixels (in black) and the entire basin (in red and blue). Numbers on the $a$ and $c$ are the mean values of GPP from three datasets over the basin. Regression equations in the upper position are for MODIS and VPM, and in the lower position are for MODIS and PML.

Figure S5 shows the comparisons of monthly and annual GPP from MODIS, VPM and PML over the entire basin during the period of 04/2002-03/2015. It is observed that at the monthly scale GPP from MODIS has the smallest ranges and VPM has the largest ranges, and PML gives higher values than the other two products. The mean monthly GPP over the basin is 119, 130 and $155 \mathrm{~mm}$ for MODIS, VPM and PML, in comparison to the mean annual values of 1431,1588 and $1901 \mathrm{~mm}$, respectively. The basinaveraged monthly GPP shares similar temporal dynamics indicated by the $R^{2}$ of 0.93 and 0.94 between MODIS and VPM and between MODIS and PML. The $R^{2}$ for monthly data of all pixels decreases to 0.75 and 0.70 respectively, still showing a good synchrony of temporal variations. For the annual data, the $R^{2}$ are higher when considering the GPP data of all pixels than the mean GPP data of the entire basin. In any case, the GPP values are highest from PML and lowest from MODIS. 